\title{
Emission of terahertz radiations from fractal antennas
}

\author{
F. Miyamaru, ${ }^{1,2, a)}$ Y. Saito, ${ }^{1}$ M. W. Takeda, ${ }^{1}$ L. Liu, ${ }^{3}$ B. Hou, ${ }^{3}$ W. Wen, ${ }^{3}$ and Ping Sheng ${ }^{3}$ \\ ${ }^{1}$ Department of Physics, Faculty of Science, Shinshu University, Asahi 3-1-1, Matsumoto, \\ Nagano 390-8621, Japan \\ ${ }^{2}$ PRESTO, Japan Science and Technology Agency, 2-1-1 Katahira, Aobaku-ku, Sendai 980-8577, Japan \\ ${ }^{3}$ Department of Physics, The Hong Kong University of Science and Technology, Clear Water Bay, Kowloon, \\ Hong Kong
}

(Received 8 October 2009; accepted 11 November 2009; published online 3 December 2009)

\begin{abstract}
We investigate the emission of terahertz radiation from a photoconductive fractal antenna fabricated on a semi-insulating gallium arsenide substrate. Owing to the self-similarity of fractal structures, our fractal antenna shows a multiband emission of terahertz radiation. The emission intensity at peak frequency is about twice that from a bow-tie antenna. We also investigate the mechanism of the multiband emission by using the finite-difference time-domain calculation. (C) 2009 American Institute of Physics. [doi:10.1063/1.3271181]
\end{abstract}

A wide variety of applications for fractal structures have been found in diverse areas of science and engineering. Among them one of the most important is on the electromagnetic dynamics. ${ }^{1}$ In particular, fractal antenna design for electromagnetic radiations ${ }^{2-4}$ is a promising application, owing to the space-filling geometric nature of some fractals ${ }^{5-7}$ with diverging surface area within a finite volume (or diverging boundary length within a finite area), and their multifrequency responses. ${ }^{8-10}$ By using the former property, there is a possibility of fabricating antennas whose size are extremely small as compared to the wavelength at the operational frequency. In addition, antennas with fractal structure can have multifrequency electromagnetic radiation emission peaks within a single unit, thus partially resolving the issue involving the emission frequency range.

In this work we study the use of fractal structure to control the emission characteristics of a terahertz radiation source. Terahertz technologies have shown remarkable development during the past decade. Since many materials exhibit distinguishing optical characteristics in the terahertz region, various practical applications have been proposed and demonstrated, e.g., medical diagnostics, ${ }^{11-13}$ security applications, $^{14-16}$ etc. Based on classical electrodynamics, the terahertz regime is the highest frequency where electromagnetic radiations can be emitted by using an electric dipole. The photoconductive antenna, which consists of a metallic antenna pattern fabricated on a semiconductor substrate, is typically used for emitting and detecting the terahertz radiation in the terahertz time domain spectroscopy (terahertz-TDS). Very simple dipole antennas, or bow-tie antennas, were mainly used as the photoconductive antenna design in the terahertz regime. ${ }^{17,18}$ There is potential to realize interesting and useful emission characteristics by adopting a more complex geometrical pattern for the photoconductive antenna designs. Some designs for photoconductive terahertz antenna were investigated for higher power output, ${ }^{19}$ better directivity, ${ }^{20}$ and other functionalities, ${ }^{21}$ e.g., polarization control.

In this paper, we report the fabrication and characterization of a photoconductive fractal antenna which emits multi-

\footnotetext{
a) Author to whom correspondence should be addressed. Electronic mail: miyamaru@shinshu-u.ac.jp.
}

band terahertz radiations. Owing to the self-similar structure of our H-fractals, our photoconductive antenna emits terahertz radiation at a few resonant frequencies. The intensity at the emission peak frequency is about twice that of a bow-tie antenna. The latter has been used in the past for terahertzTDS studies and shows relatively high emission intensities. We have also performed numerical simulations on the emission characteristics of terahertz radiation from the fractal antenna in order to obtain a more detailed understanding.

We fabricated photoconductive antennas with fractal structures on semi-insulating gallium arsenide (SI-GaAs) substrates. The inset of the Fig. 1(b) shows a micrograph of a fractal antenna used in our experiment. H-fractal patterns were generated from a $50 \mu \mathrm{m}$ thick line of length $a_{1}$, defined as the first level of the structure. The line of the second level $\left(a_{2}\right)$ is oriented along the direction perpendicular to the first level and connected at its midpoint to the end of the first level line, forming part of an $\mathrm{H}$. The third and fourth levels are constructed similarly except the line length is scaled down by a factor of $2\left(a_{3}\right.$ and $\left.a_{4}\right)$. By continuing this proce-
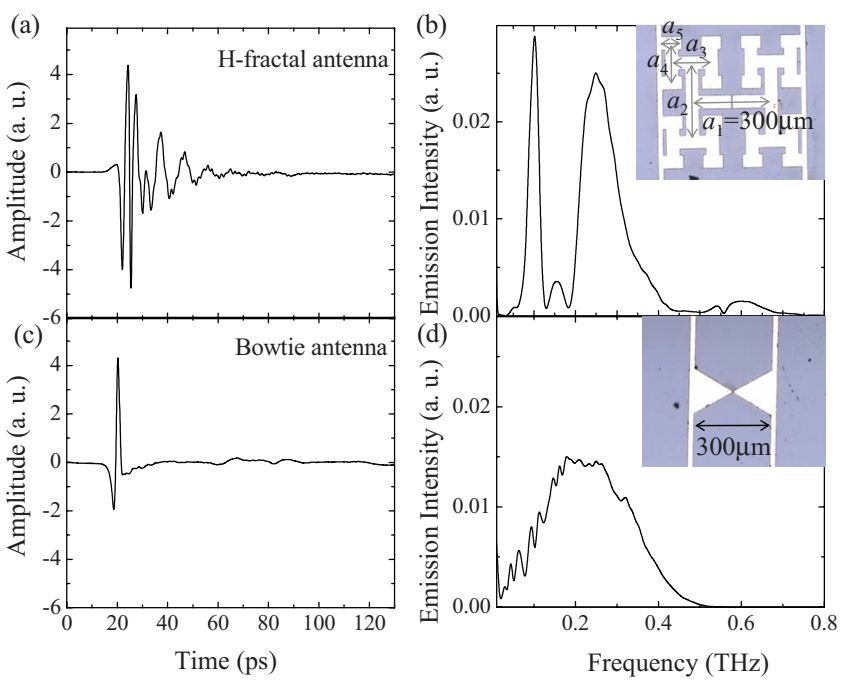

FIG. 1. (Color online) (a) Terahertz radiation generated from the fractal antenna on SI-GaAs. (b) Fourier-transformed power spectrum of (a). (c) Terahertz radiation generated from the bow-tie type antenna. (d) Fouriertransformed power spectrum of (c). Inset of (b) and (c) show photographs of the fractal and bow-tie type antenna, respectively. 
dure, a self-similar $\mathrm{H}$-fractal structure is realized. In our experiment, the fractal pattern consists of five levels. The $5 \mu \mathrm{m}$ wide gap is fabricated at the center of the line of the first level for exciting the transient current by illuminating the femtosecond laser pulse.

The emission spectra of terahertz radiations were measured by the terahertz -TDS. ${ }^{22}$ Terahertz radiations were emitted from a photoconductive fractal antenna illuminated by a $120 \mathrm{fs}$ laser pump pulses at $800 \mathrm{~nm}$. The emitted terahertz radiation was collimated and focused with a pair of off-axis paraboloidal mirrors, and detected by a dipole-type photoconductive antenna gated with time-delayed probe laser pulses, diverted from the pump pulses. By Fourier transform, the measured wave form of the terahertz radiation was converted into power intensity spectrum. The detected polarization of the terahertz wave is parallel to the line of the first level of the fractal structure.

Figure 1(a) shows the measured terahertz waveform emitted from the photoconductive $\mathrm{H}$-fractal antenna with five levels. The line length of the first level is $a_{1}=300 \mu \mathrm{m}$. Two components of the terahertz waves with different frequencies are clearly seen at around $t=20 \mathrm{ps}$ and $40 \mathrm{ps}$. This means that two resonant modes in the fractal structure contribute to the emission of the terahertz wave. As the result of the interference between these two components, the beat can be observed clearly at around $35 \mathrm{ps}$ in the terahertz waveform. The multiple resonant behavior of the fractal antenna is also observed in the Fourier spectrum as shown in Fig. 1(b). The lower emission peak (mode 1) is located at $0.10 \mathrm{THz}$ and higher emission peak (mode 3 ) is located at $0.25 \mathrm{THz}$. In addition, the third weak peak (mode 2) can be observed at around $0.16 \mathrm{THz}$. The characteristic of multiple-peak emission originates from the multiple resonances intrinsic to the $\mathrm{H}$-fractal structure that were observed previously. ${ }^{23,24}$ For comparison, we have also measured the terahertz wave emitted from a bow-tie type photoconductive antenna. The wave form and emission spectrum of the bow-tie type antenna are shown in Figs. 1(c) and 1(d). In contrast to the H-fractal antenna, the wave form from the bow-tie type antenna consists of only one cycle pulse. The emission spectrum shows one broad peak, in contrast to that from the H-fractal antenna. Interestingly, the highest peak intensity of the fractal antenna emission spectrum is almost twice that of the bowtie antenna. This is due to the strong resonant modes in the $\mathrm{H}$-fractal structure.

In order to investigate the time evolution of the terahertz wave emitted from the $\mathrm{H}$-fractal antenna, we applied a short time Fourier transform (STFT) to the measured wave form of Fig. 1(a). In this analysis, we used the Gaussian function as a variable time window. By changing the delay time between the measured wave form and the Gaussian time window, we calculate the Fourier spectrum at each delay time. In this analysis, the window width $\delta$ of the Gaussian time window determines both the time and frequency resolutions of the STFT spectrum. The time resolution increases proportionally with decreasing $\delta$, while the frequency resolution decreases with decreasing $\delta$. In order to obtain the appropriate time and frequency resolutions, we select the window width to be $\delta$ $=34 \mathrm{ps}$ in this analysis. The temporal evolution of the emission spectra derived by STFT analysis is shown in Fig. 2. The horizontal and vertical axes of the figure show the delay time of the Gaussian time window and frequency, respectively. The Fourier intensity of the STFT analysis is pre-

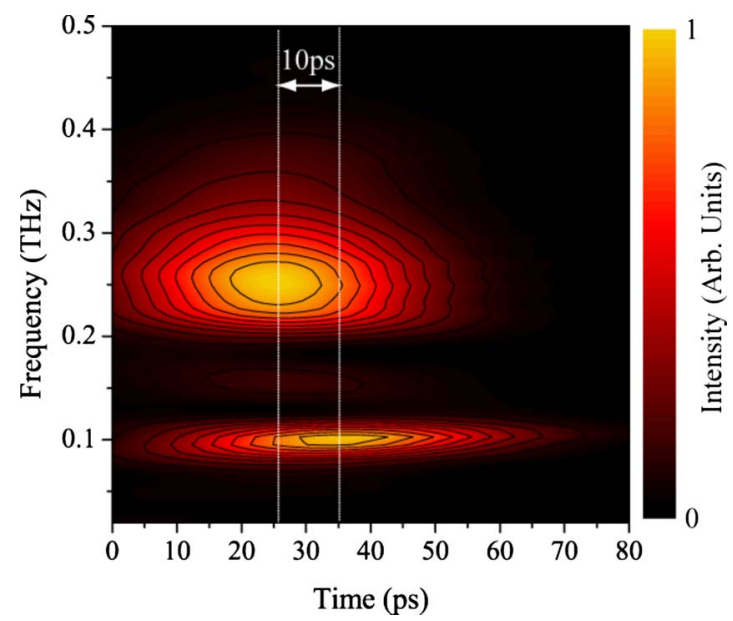

FIG. 2. (Color online) Fourier-transformed spectrum of Fig. 1(a) with the variable Gaussian time window. The horizontal axis indicates the delay time between measured waveform and Gaussian time window with the window width $\delta=34$ ps.

sented as a color scale, normalized by the highest intensity of the STFT spectrum. It should be noted that the appearance time of the peak of mode 1 is delayed for $10 \mathrm{ps}$ as compared to that of mode 3 . This shows the difference in the generation time of the terahertz wave among the different resonant modes.

To investigate the mechanism of terahertz wave emission from the fractal antenna in more detail, we have performed the numerical simulations by using the finite difference time domain (FDTD) approach. Figure 3(a) shows a comparison between the measured emission spectrum and FDTD simulations. The fractal antenna used in Fig. 3(a) is same as that in Fig. 1(a). In the FDTD simulations, the semi-insulating
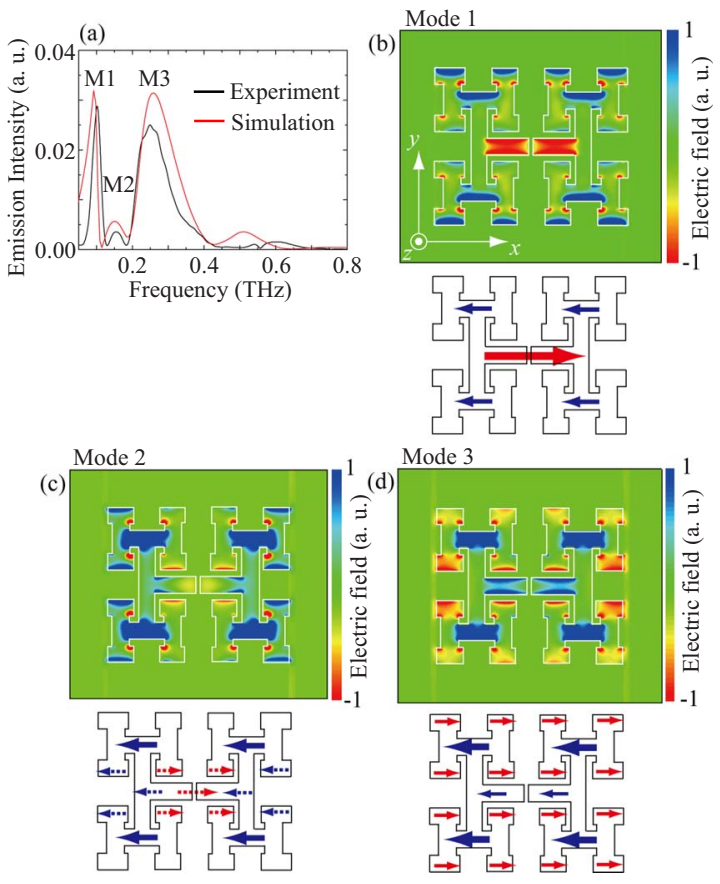

FIG. 3. (Color online) (a) Measured (black line) and calculated (red line) emission spectra generated from the fractal antenna. [(b)-(d)] Calculated electric field distributions for a negative type fractal structure at the resonant frequency of modes 1,2 , and 3 , respectively. The polarization of the electric field is along to the $y$ direction. Bottom pictures of Figs. 3(b)-3(d) are schematic pictures of current estimated from electric field distributions. 
GaAs substrate is not included and the metallic antenna structure is surrounded by air. In this case, the frequency of the resonant peak shifts to the higher frequency as compared to that of experiment where the semi-insulating GaAs substrate is present. The effect of the GaAs substrate is compensated by multiplying the frequency by the effective refractive index $n_{\text {eff }} 18$

$$
n_{\mathrm{eff}}=\sqrt{\frac{1+\varepsilon_{\mathrm{GaAs}}}{2}},
$$

where $\varepsilon_{\mathrm{GaAs}}$ is permittivity of GaAs substrate $\left(\varepsilon_{\mathrm{GaAs}} \sim 13\right)$. A Good agreement between experiment and FDTD calculations is observed in Fig. 3(a). The calculated spatial distributions of the electric field for modes 1, 2, and 3 are shown in Figs. 3(b)-3(d), respectively. These simulations were performed for the complementary type (negative type) of the fractal structure where the fractal structure is perforated on the perfect conductor sheet. The observed polarization of the electric field is along $y$ direction. The observed position in $\mathrm{z}$ direction is the center of the fractal pattern. Since the perfect conductor is used in our simulations, it is not possible to observe the electromagnetic field distribution inside the positive type fractal pattern. Therefore, we cannot calculate the current distribution in the fractal pattern. To obtain the information about the current distribution, we used negative type of fractal structure. Based on Babinet's principle, these calculated results for the negative type of fractal structure correspond to the $x$ polarization of the electric field for the positive type of the fractal structure used in our experiment. In Fig. 3(b), a strong localization of the electric field is observed at the line of the first level. A relatively weaker localization of the electric field is also observed at the line of the third level with the reversed sign with respect to that of the first level due to the delay of current flows. The positive and negative signs denote the direction of the electric field. The schematic picture of the current distribution on the fractal antenna estimated from the calculated result is shown at the bottom of Fig. 3(b). It is clear that the resonant emission of the mode 1 results mainly from the currents flowing along lines of the first and third levels (with counter flowing directions). Similar current distribution is observed for mode 3 as shown in Fig. 1(d). For mode 3, the electric field is localized most strongly at lines of the third level. Relatively weaker localization is observed at the line of the first level. Contrary to mode 1 , the signs of the electric field localized at the first and third levels are the same for mode 3 . The localization of the counter direction of electric fields is observed at lines of the fifth level. As a result, the current distribution on the fractal antenna can be estimated as shown in the bottom of Fig. 3(d). These current distributions for modes 1 and 3 clearly show fractal characteristics of the $\mathrm{H}$ fractal photoconductive antenna.

The electric field distribution for mode 2 [Fig. 3(c)] is completely different from those of modes 1 and 3 . For mode 2 , the electric field is localized most strongly at lines of the third level, which is similar to mode 3. However, the electric fields localized at lines of the first and fifth level show dis- tinct distributions. The estimated current distribution is shown in the bottom of Fig. 3(c). At the first level, the current flows rightward around the center of the line. Around both edges of the line, however, the current flows leftward. These counter directions of the current flow in the first level bring the result of the cancellation of an electromagnetic wave emission. Similar cancellations occur at lines of fifth level where the current flows of counter directions appear at nearest neighbor lines of the fifth level. The origin of the low intensity of the emission for mode 2 can be attributed to such cancellation processes of an electromagnetic wave emission in the fractal structure.

This work was supported partially by the Ministry of Education, Science, Sports and Culture, Grant-in-Aid for Scientific Research (A) No. 20244047, and for Young Scientists (B) No. 21760036. F. M. is also supported by the Murata Science Foundation.

${ }^{1}$ D. L. Jaggard, in Recent Advances in Electromagnetic Theory, edited by H. N. Kritikos and D. L. Jaggard (Springer, New York, 1990), p. 183.

${ }^{2}$ A. Fujimoto, A. Henderson, K. Hirasawa, and J. R. James, Small Antennas (Wiley, New York, 1978).

${ }^{3}$ S. Maci and G. Biffi Gentili, IEEE Antennas Propag. Mag. 39, 13 (1997).

${ }^{4}$ D. H. Werner, R. L. Haupt, and P. L. Werner, IEEE Antennas Propag. Mag. 41, 37 (1999).

${ }^{5}$ J. Anguera, C. Puente, C. Borja, R. Montero, and J. Soler, Microwave Opt. Technol. Lett. 31, 239 (2001).

${ }^{6}$ J. Anguera, C. Puente, E. Martinez, and E. Rozan, Microwave Opt. Technol. Lett. 36, 102 (2003).

${ }^{7}$ B. Mirzapour and A. A. L. Neyestanak, Microwave Opt. Technol. Lett. 49, 1077 (2007).

${ }^{8}$ X. Liang and M. Y. W. Chi, Microwave Opt. Technol. Lett. 23, 242 (1999).

${ }^{9}$ S. R. Best, Microwave Opt. Technol. Lett. 35, 371 (2002).

${ }^{10}$ K. Siakavara and F. Tsaidaris, Microwave Opt. Technol. Lett. 41, 180 (2004).

${ }^{11}$ K. J. Siebert, H. Quast, R. Leonhardt, T. Löffler, M. Thomson, T. Bauwe, H. G. Roskos, and S. Czasch, Appl. Phys. Lett. 80, 3003 (2002).

${ }^{12}$ S. Nakajima, H. Hoshina, M. Yamashita, C. Otani, and N. Miyoshi, Appl. Phys. Lett. 90,041102 (2007).

${ }^{13}$ H. Hoshina, A. Hayashi, N. Miyoshi, F. Miyamaru, and C. Otani, Appl. Phys. Lett. 94, 123901 (2009).

${ }^{14}$ K. Yamamoto, M. Yamaguchi, F. Miyamaru, M. Tani, and M. Hangyo, Jpn. J. Appl. Phys., Part 2 43, L414 (2004).

${ }^{15}$ N. Karpowicz, H. Zhong, C. Zhang, K.-I. Lin, J.-S. Hwang, J. Xu, and X.-C. Zhang, Appl. Phys. Lett. 86, 054105 (2005).

${ }^{16}$ H.-B. Liu, Y. Chen, G. J. Bastiaans, and X.-C. Zhang, Opt. Express 14, 415 (2006).

${ }^{17}$ M. van Exter, C. Fattinger, and D. Grischkowsky, Appl. Phys. Lett. 55, 337 (1989).

${ }^{18}$ M. Tani, S. Matsuura, K. Sakai, and S. Nakashima, Appl. Opt. 36, 7853 (1997).

${ }^{19}$ R. Mendis, C. Sydlo, J. Sigmund, M. Feiginov, P. Meissner, and H. L. Hartnagel, Solid-State Electron. 48, 2041 (2004).

${ }^{20}$ M. Awad, M. Nagel, H. Kurz, J. Herfort, and K. Ploog, Appl. Phys. Lett. 91, 181124 (2007).

${ }^{21}$ Y. Hirota, R. Hattori, M. Tani, and M. Hangyo, Opt. Express 14, 4486 (2006).

${ }^{22}$ M. Hangyo, T. Nagashima, and S. Nashima, Meas. Sci. Technol. 13, 1727 (2002).

${ }^{23}$ W. Wen, L. Zhou, J. Li, W. Ge, C. T. Chan, and P. Sheng, Phys. Rev. Lett. 89, 223901 (2002).

${ }^{24}$ F. Miyamaru, Y. Saito, M. W. Takeda, B. Hou, L. Liu, W. Wen, and P. Sheng, Phys. Rev. B 77, 045124 (2008). 